\title{
The effect of programmed intermittent epidural bolus compared with continuous epidural infusion in labor analgesia with ropivacaine: a meta-analysis of randomized controlled trials
}

\author{
Rui Huang ${ }^{1 \# \wedge}$, Jiang Zhu ${ }^{2 \# \wedge}$, Zizuo Zhao ${ }^{1}$, Bin Wang ${ }^{1 \wedge}$ \\ ${ }^{1}$ Department of Anesthesiology, The First Affiliated Hospital of Chongqing Medical University, Chongqing, China; ${ }^{2}$ Department of Endocrine and \\ Breast Surgery, The First Affiliated Hospital of Chongqing Medical University, Chongqing, China \\ Contributions: (I) Conception and design: R Huang, J Zhu, B Wang; (II) Administrative support: B Wang; (III) Provision of study materials or \\ patients: R Huang, J Zhu, B Wang; (IV) Collection and assembly of data: R Huang, J Zhu; (V) Data analysis and interpretation: R Huang, J Zhu, B \\ Wang; (VI) Manuscript writing: All authors; (VII) Final approval of manuscript: All authors. \\ "These authors contributed equally to this work. \\ Correspondence to: Bin Wang. Department of Anesthesiology, The First Affiliated Hospital of Chongqing Medical University, 1 Youyi Rd, Chongqing \\ 400016, China. Email: 13637815096@163.com.
}

Background Programmed intermittent epidural bolus (PIEB) as a new technique for labor analgesia has aroused extensive attention. The character of separation of the motor block to sensory block makes ropivacaine becoming an important local anesthetic for labor analgesia. In this meta-analysis, we aimed to assess the efficiency and safety of PIEB regime compared to continuous epidural infusion (CEI) regime on labor analgesia with ropivacaine following the evidence emerged newly.

Methods: PubMed, EMBASE and the Cochrane library were searched for potential articles. Eligible studies should meet these criterions: (I) healthy women; (II) it should compare PIEB and CEI; (III) ropivacaine should be use as local anesthetic for the maintenance of analgesia; (IV) the study should report the any of the outcomes we need. Maternal satisfaction, consumption of ropivacaine and duration of labor as well as the adverse effect were used to measure the efficacy and safety of those two regimes. Mean difference (MD), relative risk (RR), 95\% confidence intervals (CI) were used to present the final results.

Results: Ten articles of randomized controlled trials and 3,790 subjects were eventually included in study. The pooled results showed that PIEB with ropivacaine significantly improved satisfaction (MD, 7.87; 95\% CI: 6.02 to $9.72 ; \mathrm{I}^{2}=0 \% ; \mathrm{P}<0.001$ ), reduced the local anesthetic (milligram) in total ( $\mathrm{MD},-10.37$ milligrams; 95\% CI: -17.70 to $\left.-3.03 ; \mathrm{I}^{2}=94 \% ; \mathrm{P}<0.001\right)$ and hourly (MD, -1.80 milligrams; $95 \% \mathrm{CI}:-2.62$ to -0.98 ; $\left.\mathrm{I}^{2}=56 \% ; \mathrm{P}<0.001\right)$. PIEB shortened the second stage of labor but has similar total duration of labor and it also decrease the incidence of motor block compare to CEI. There were no differences in mode of delivery and rescue bolus between two groups.

Conclusions: This study shows that PIEB regime was associated with higher satisfaction, lower consumption of ropivacaine in hours and totally, and shorter duration of second stage of labor compared to CEI in analgesia with ropivacaine during childbirth. PIEB regime has greater safety on fetus and maternity than CEI regime and it decreased the incidence of motor block without increasing other side effects compared to CEI.

Keywords: Programmed intermittent epidural bolus (PIEB); continuous epidural infusion (CEI); ropivacaine; labor analgesia; meta-analysis

Submitted Aug 03, 2020. Accepted for publication Nov 17, 2020.

doi: 10.21037/apm-20-1541

View this article at: http://dx.doi.org/10.21037/apm-20-1541

^ ORCID: Rui Huang, 0000-0002-3342-135X; Jiang Zhu, 0000-0003-4194-2355; Bin Wang, 0000-0002-9177-2975. 


\section{Introduction}

Labor pain is one of the most painful experiences of every mother (1). Severe acute pain during labor is an important incentive of postpartum persistent pain which may have serious interference with a number of women's daily life (2). Epidural analgesia is one of the most safe and effective methods for labor analgesia, providing effective pain relief during labor and may decrease the occurrence of cesarean delivery (3-5). and it can be administered in different ways. Continuous epidural infusion (CEI) with or without patient-controlled epidural analgesia (PCEA) has been the one of the most common analgesic techniques we used, which can effectively achieve adequate pain relief and reduce discomfort, and it also becomes a standard of labor epidural analgesic in North America and Europe in recent decades (6). However, CEI may increase the rate of dystocia and instrumental delivery, and may result in a higher incidence of motor blockade and lower satisfaction compared to traditional systemic analgesia (7). With the development of computer technology, programmed intermittent epidural bolus (PIEB) is a new automated method of administering epidural solutions at a fixed bolus and scheduled interval, which used as an alternative to CEI alone or as a background administration with the PCEA technique (8). It also has potential advantages of better spread of local anesthetic in the epidural space which made it has greater sensory blockade compared CEI (9). PIEB may cover the shortages of CEI program, improve satisfaction. It is not clear that whether PIEB can replace CEI completely in labor analgesia.

As one of the most important long-acting amide local anesthetics (10), ropivacaine is commonly used for labor analgesia. Compared with bupivacaine, ropivacaine has been associated with less central nervous system and cardiac toxicity (11). Evidence suggests that ropivacaine results in greater sensory and less motor block than does bupivacaine, although their relative potencies differ. Ropivacaine increases analgesic effect without increasing the degree of motor block. Because of that, ropivacaine has attracted increased attention for epidural analgesia on labor analgesia in recent years. However, some articles recommended CEI regime while opponent argue that PIEB has better efficacy and safety. It is not clear yet which is better for maternity in pain relief when using ropivacaine. Therefore, we conducted this study in order to investigate the difference in maternal satisfaction, consumption of ropivacaine (primary outcomes) as well as duration of labor and other adverse effects (second outcomes) between PIEB and CEI in the labor anesthesia with ropivacaine.

We present the following article in accordance with the PRISMA reporting checklist (available at http://dx.doi. org/10.21037/apm-20-1541).

\section{Methods}

\section{Search strategy}

The literature search was conducted across the electronic databases including PubMed, Embase and Cochrane Library from the establishment of the database in February 2020, using the keywords "ropivacaine, intermittent, epidural, Labor, randomized" as well as relevant synonyms. The details of search strategy were presented in Appendix 1. In addition, we also searched the reference lists of relevant studies by hand to identify other studies meeting the inclusion criteria.

\section{Inclusion and exclusion criteria (study selection)}

Two investigators screened the potential eligible articles independently by scanning the titles and abstracts. The duplicates, reviews, case reports, and letters were excluded. Then, we read the full article of the rest studies to sort out the ones that did not meet the inclusion criteria. A third reviewer consulted with the two investigators if there was any divergence between them. Eligible studies were present as follows: (I) we included RCTs only; (II) the study should compare PIEB and CEI with or without PCEA; (III) ropivacaine should be use as local anesthetic with or without opioid for maintenance of labor epidural analgesia; (IV) all women should be in healthy conditions. Whether combining spinal-epidural analgesia or not were included. These studies could be excluded if the authors had not clearly described the protocols for maintaining labor analgesia and the methods of PIEB regime, CEI regime (such as specific dose, concentration, speed, etc.).

\section{Quality assessment}

Two independent authors evaluated the quality of all eligible studies by using Cochrane Collaboration's tool (12) for assessing the risk of bias, which assess quality of studies according following aspects: randomized sequence generation, allocation concealment, blinding, incomplete data, selective reporting, and other potential bias. Each of 
the ten trials was assessed as low risk (+), unclear risk (?), or high risk (-) which represent low, moderate, and high quality of including studies respectively. The final decision was made by consulting with another investigator if any disagreement occurred.

\section{Data extraction}

Data was extracted from the inclusion studies as following information: characteristics included first author, year of publication, country, and analgesia protocols. Primary outcomes included maternal satisfaction (estimating by verbal rating scale (VRS) from 0 (presenting very dissatisfied) to 100 (presenting extremely satisfied), the consumption of ropivacaine (total consumption and the dose delivered per hour) and duration of labor (total duration and second stage of duration). Secondary outcomes included motor block, mode of delivery (operative vaginal, cesarean delivery) and rescue anesthesia (patients who need additional pain relief by anesthetist). Other safety outcomes included hypotension and nausea-vomiting and fetal bradycardia.

When the data were not reported directly, we obtained the consumption of ropivacaine per hour by converting the total dose of ropivacaine and the mean duration of epidural analgesia presented in studies (6). If the data was expressed as medians, ranges, and confidence intervals (CIs) in article, the mean and standard deviations were calculated from extracted data according to Hozo et al. (13). Differences was settled by discussion or decided by the third author.

\section{Statistical analysis}

All data we extracted were tabulated in Microsoft Excel. All statistical analysis was performed using Review Manager 5.3. For continuous parameters, pooled data were present as mean difference (MD) with $95 \%$ confidence intervals (CI). And the relative risk (RR) was applied to dichotomous variables. Heterogeneity across the studies were assessed using the $\mathrm{Q}$ statistic and the $\mathrm{I}^{2}$ statistic. $\mathrm{P}$ values $<0.1$ (Q test) or $\mathrm{I}^{2}>50 \%$ suggested that there is significant heterogeneity. When $\mathrm{I}^{2} \leq 50 \%$ or $\mathrm{P}$ values $>0.1$, we use the fixed effect model to combine the data and the random effect model was applied when $\mathrm{I}^{2}>50 \%$ or $\mathrm{P}$ values $<0.1$. Publication bias were assessed by visual examination of funnel plots. We considered the combined results are statistically significant when $\mathrm{P}$ value $<0.05$.

\section{Result}

\section{Literature search}

Initially, the literature search yielded 93 potential studies. There remained 56 articles after removing duplicates. We screened the abstracts of remained 56 articles and irrelevant articles were excluded, of which 25 full-texts were reviewed. Finally, ten of those articles were included in this meta-analysis (14-23). The flow diagram was presented in Figure 1.

\section{Characteristics of the included studies}

Ten articles were identified to this meta-analysis. All 3,790 participants were included in present articles; 1,894 of whom were accepted PIEB while 1,896 use CEI regime. First author, year of publication, regime design was present in Table 1. All ten included trials were classified as low to quality. The risk of bias was concluded in Figure 2.

\section{Primary outcomes}

\section{Maternal satisfaction}

Five of the studies reported maternal satisfaction. One of the studies used a score from 0 (not satisfied at all) to 10 (completely satisfied) to evaluate satisfaction (22). Higher maternal satisfaction was observed in PIEB group compared to $\mathrm{CEI}$ in this study. Other researches evaluated satisfaction using verbal rating scale (VRS) from 0 to 100 (0 presenting very dissatisfied and 100 presenting extremely satisfied). The pooled data following other four studies [using verbal numeric scale (VNS) from 0 to 100] were statistically significant with no heterogeneity (MD, 7.87; 95\% CI: 6.02 to $9.72 ; \mathrm{I}^{2}=0 \% ; \mathrm{P}<0.001 ;$ Figure 3$)$.

\section{Dose of ropivacaine}

The concentration and the regimen of ropivacaine for every trail was present in Table 1 . The data of total dose of ropivacaine was present in seven studies including 3,454 participants (15-17,19,21-23). And the dose of ropivacaine per hour were reported in five studies $(14,15,18,19,22)$. The result indicated that the total dose of ropivacaine was lower in PIEB group compared with CEI group but with significant heterogeneity among those studies by pooling analysis (MD, -10.37 milligrams; 95\% CI: -17.70 to -3.03 ; $\mathrm{I}^{2}=94 \% ; \mathrm{P}<0.001 ;$ Figure $4 A$ ). Therefore, a subgroup analysis was performed, and heterogeneity was not found by using 


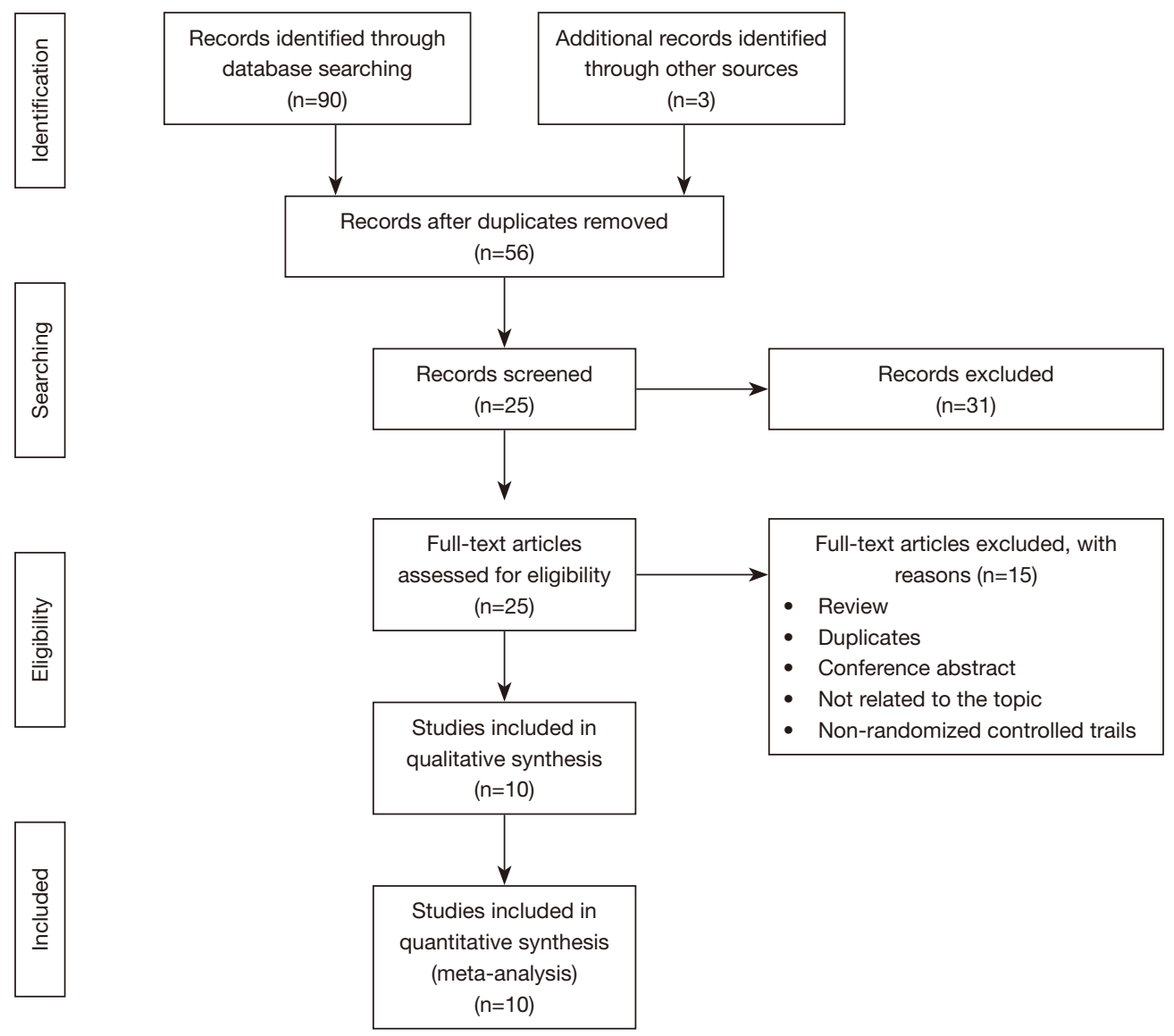

Figure 1 Flow diagram of study selection (PRISMA).

combined spinal anesthesia (CSE) for labor anesthesia while great heterogeneity was still observed by epidural anesthesia (EA). For ropivacaine delivered per hour, the result was consistent with the result of total dose. The consumption of ropivacaine per hour in PIEB group had 1.08 milligrams less than in CEI group (MD, -1.80 milligrams; $95 \% \mathrm{CI}$ : -2.62 to $-0.98 ; \mathrm{I}^{2}=56 \% ; \mathrm{P}<0.001 ;$ Figure $\left.4 B\right)$.

\section{Duration of labor}

Five of the studies reported the total duration of labor $(14,15,17,18,21)$. There were not statistically significant when data were pooled from the five trials (MD, -12.67 minutes; $95 \%$ CI: -47.95 to $22.60 ; \mathrm{I}^{2}=28 \%$; $\mathrm{P}=0.48$; Figure $5 A$ ). Eight of the studies offered the information of duration of second stage of labor (14-19,22,23). The pooled results were statistically significant which indicated that there was a shorter duration of second stage of labor in PIEB group than CEI group (MD, -5.62 minutes; $95 \%$ CI: -9.81 to $-1.43 ; \mathrm{I}^{2}=67 \% ; \mathrm{P}=0.009 ;$ Figure $\left.5 B\right)$.

\section{Secondary outcomes}

\section{Motor block}

Motor block was assessed by Bromage score (24). Five articles reported the number of people who experienced motor blocks $(14,15,20,21,23)$. And we observed that the occurrence of motor block in PIEB group was lower compared it in CEI group with no heterogeneity (RR, 0.58; $95 \%$ CI, 0.35-0.98; $\mathrm{I}^{2}=0 \%$; $\mathrm{P}=0.04$; Figure 6).

\section{Mode of delivery}

Eight trials present the results of the mode of deliveries. A total of 3,478 subjects were included in the analysis. The summarized analysis indicated that there is no difference in operative vaginal rate (RR, $0.87 ; 95 \%$ CI, $0.69-1.09 ; \mathrm{I}^{2}=0 \%$; $\mathrm{P}=0.22$; Figure $7 A$ ). For cesarean delivery, the pooled data also showed no difference (RR, 0. 96; 95\% CI, 0.69-1.32; $\mathrm{I}^{2}=0 \% ; \mathrm{P}=0.80 ;$ Figure $7 B$ ). This all suggested that $\mathrm{PIEB}$ group may not have a reduction in the rates of those two types of delivery. 


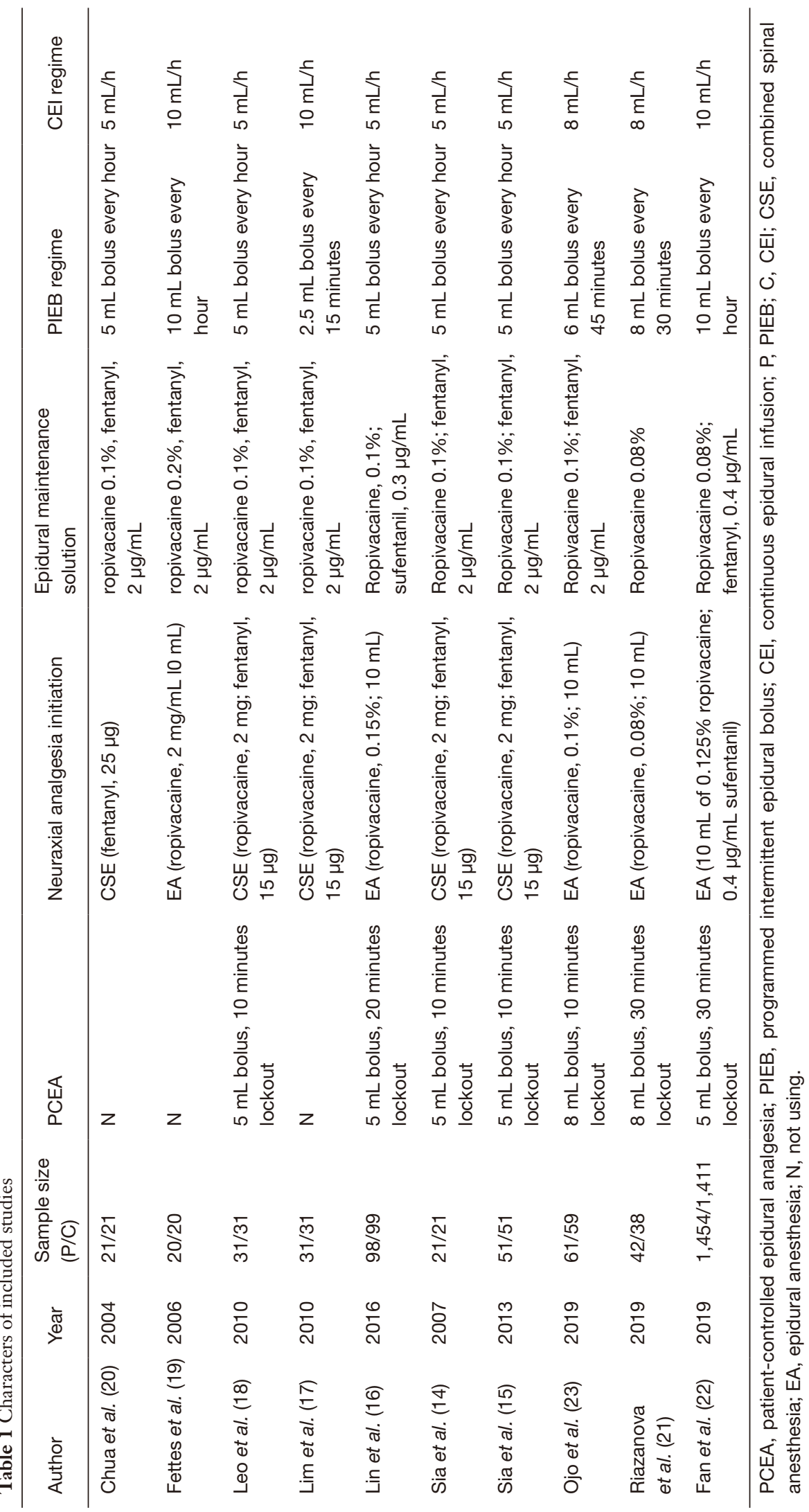




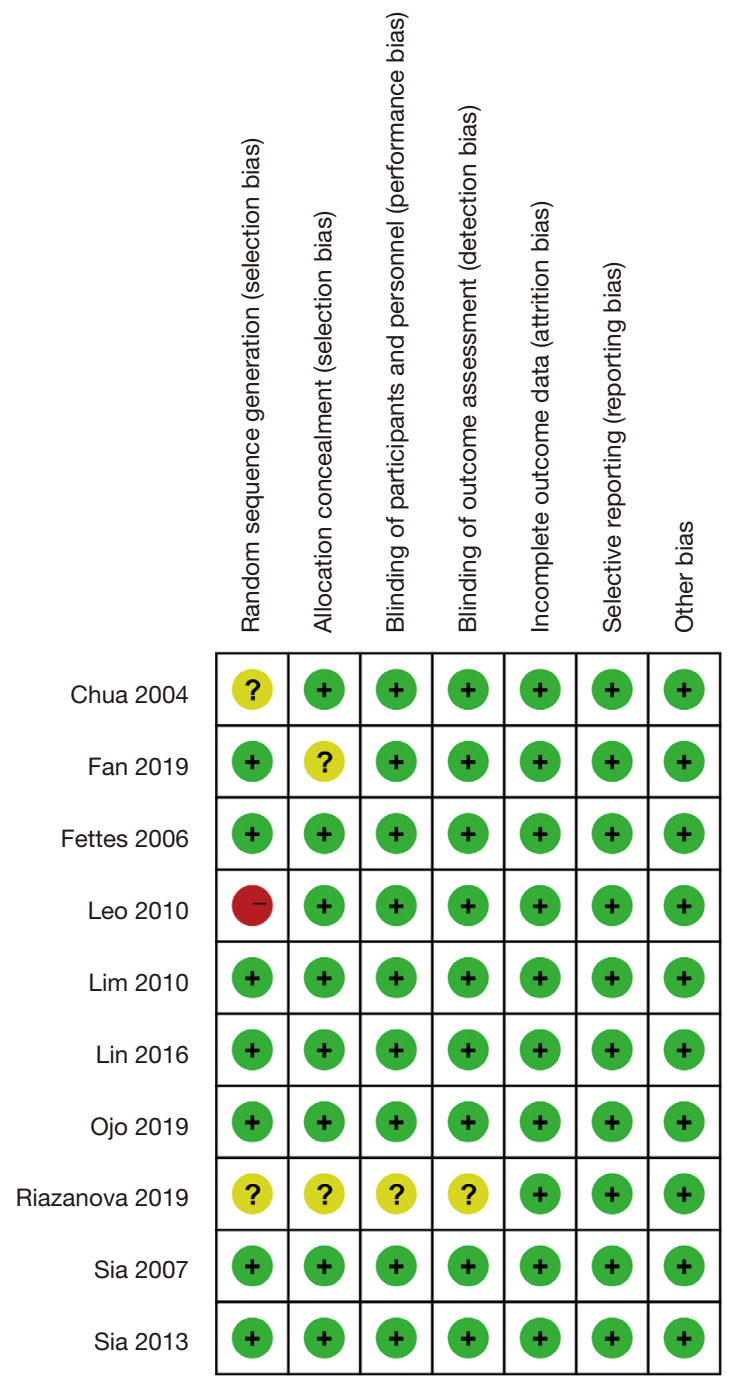

Figure 2 The risk of bias summary; low risk (+), unclear risk (?), or high risk (-).

\section{Rescue analgesia}

Seven trials reported the events for the need of additional rescue analgesia by anesthetist $(14,15,17-20,23)$. Only two of these studies had a reduction on the need of additional rescue bolus $(15,19)$. The summary data on the rescue analgesia was not statistically significant (RR, 0.78; 95\% CI, $0.50-1.21 ; \mathrm{I}^{2}=54 \% ; \mathrm{P}=0.27$; Figure 8).

\section{Additional outcomes}

Apgar scores at one minute were offered by five articles $(16,19,21-23)$. There were not significant differences in any of those trials. Eight of articles showed the data of
Apgar scores at five minutes (15-19,21-23). There was not any significance difference between the two groups. The all averages of Apgar scores were surpassed the score of seven in two of the groups which may indicate a safety effect for fetus. The study conducted by Sia divided the participants by the score of seven (14). Majority of people in both groups scored more than seven points (17/21 in PIEB group; 19/21 in CEI group; RR, 0.89; $\mathrm{P}=0.38$ ).

Other adverse outcomes were also extracted, which were listed in Table 2 including nausea, vomiting, hypotension, and fetal bradycardia. Statistic difference was not observed in the pooled analysis of these side effects of parturient.

\section{Publication bias}

We assessed the probability of publication bias of included studies by visual examination of funnel plots. The funnel plot was symmetrical suggesting that there was no possibility of publication bias (Figure S1).

\section{Discussion}

This meta-analysis of 10 RCTs compared the effect and safety of PIEB and CEI on maternity and fetus by using ropivacaine as local anesthetic. The meaningful finding of the meta-analysis demonstrated that as the background infusion, PIEB regime with ropivacaine significantly improve mother's satisfaction, reduced the consumption of ropivacaine in hours and total, shortened the second stage of labor without increasing the total duration of labor and reduced the incidence of motor block. But no differences were found with regard to the occurrence of instrumental and cesarean delivery, need of rescue bolus. There were similar incidences of those adverse events between two groups (fetal bradycardia, nausea, vomiting, hypotension). All including studies were considered as low risk of bias.

Maternal satisfaction is a multidimensional evaluation which is affected by all care provided during childbirth such as the length of labor, degree of pain, side effect, etc (25). To our knowledge, the conventional definition of maternal satisfaction is using a score of 0 (very dissatisfied) to 100 or 10 (very satisfied) by patient's verbal reporting or visual analogue scale (26). Verbal numeric scale (VNS) from 0 (not satisfied at all) to 10 (completely satisfied) is also frequentlyused in measuring satisfaction. A study conducted by Fan (22) used a score from 0 (not satisfied at all) to 10 (completely satisfied) to evaluate satisfaction and found that PIEB markedly improved maternal satisfaction compared 


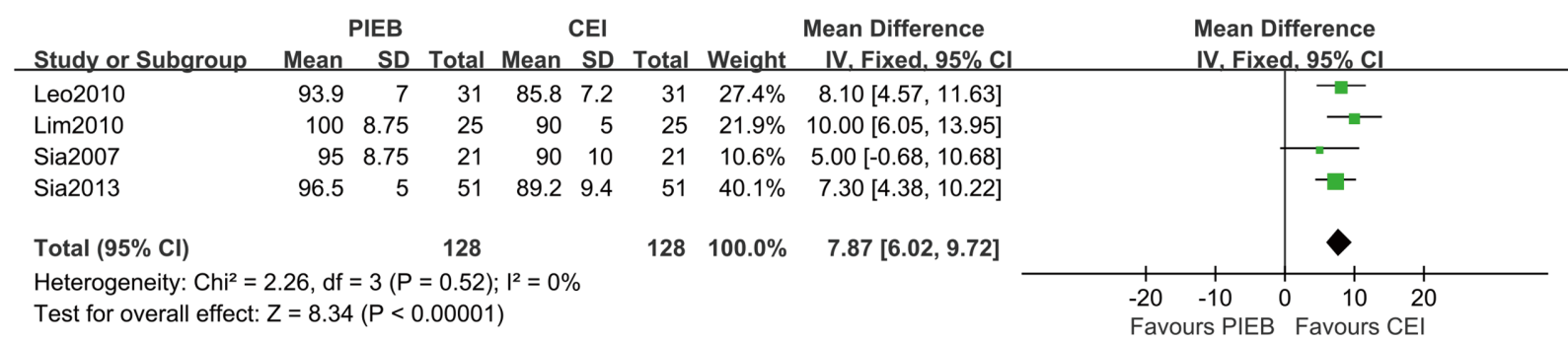

Figure 3 Forest plot of maternal satisfaction. PIEB, programmed intermittent epidural boluses; CEI, continuous epidural infusion.

A

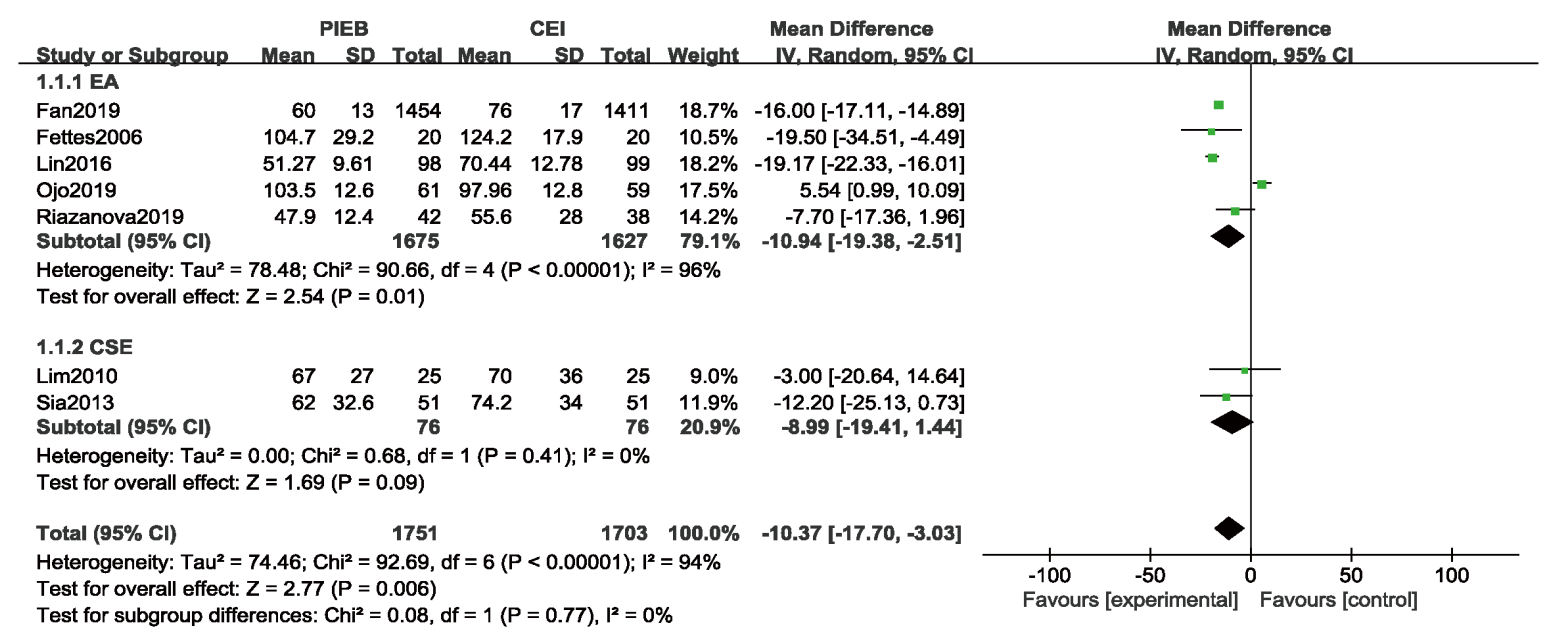

B

\begin{tabular}{|c|c|c|c|c|c|c|c|c|c|c|c|c|}
\hline Study or Subgroup & Mean & $\begin{array}{l}\text { IEB } \\
\text { SD }\end{array}$ & Total & Mean & $\begin{array}{c}\text { CEI } \\
\text { SD }\end{array}$ & Total & Weight & $\begin{array}{l}\text { Mean Difference } \\
\text { IV, Random, } 95 \% \mathrm{Cl}\end{array}$ & & $\begin{array}{l}\text { Mean Difi } \\
\text { IV, Randor }\end{array}$ & $\begin{array}{l}\text { fference } \\
\mathrm{m}, 95 \% \mathrm{cl}\end{array}$ & \\
\hline Fan2019 & 9.4 & 2 & 1454 & 11.9 & 2.7 & 1411 & $40.3 \%$ & $-2.50[-2.67,-2.33]$ & & 口 & & \\
\hline Fettes2006 & 25 & 7 & 20 & 27 & 3.9 & 20 & $4.8 \%$ & $-2.00[-5.51,1.51]$ & & & & \\
\hline Leo2010 & 7.6 & 3.2 & 31 & 9.3 & 2.5 & 31 & $18.2 \%$ & $-1.70[-3.13,-0.27]$ & & & & \\
\hline Sia2007 & 6.5 & 3.4 & 21 & 7.5 & 2 & 21 & $15.0 \%$ & $-1.00[-2.69,0.69]$ & & & & \\
\hline Sia2013 & 10 & 3 & 51 & 11.1 & 3.2 & 51 & $21.7 \%$ & $-1.10[-2.30,0.10]$ & & & & \\
\hline Total $(95 \% \mathrm{Cl})$ & & & 1577 & & & 1534 & $100.0 \%$ & $-1.80[-2.62,-0.98]$ & & & & \\
\hline \multicolumn{9}{|c|}{$\begin{array}{l}\text { Heterogeneity: } \text { Tau }^{2}=0.43 ; \mathrm{Chi}^{2}=9.10, \mathrm{df}=4(P=0.06) ; I^{2}=56 \% \\
\text { Test for overall effect: } Z=4.31(P<0.0001)\end{array}$} & -10 & $\begin{array}{l}-5 \\
\text { vours PIEB }\end{array}$ & Favours $\stackrel{5}{\mathrm{CE}}$ & 10 \\
\hline
\end{tabular}

Figure 4 Forest plots of the consumption of ropivacaine. (A) Forest plot of the total consumption of ropivacaine (milligram); (B) forest plot of the usage of ropivacaine hourly (milligram). CSE, combined spinal anesthesia; EA, epidural anesthesia; PIEB, programmed intermittent epidural boluses; CEI, continuous epidural infusion.

to CEI group. The pooled result of other four studies also shows a consistent consequence. The factors related to maternal satisfaction are having expectations for labor and delivery (27). It is possible that PIEB may associated with less uncomfortable feeling and better anesthesia. That could be one of the main reasons why PIEB improve satisfaction. Therefore, PIEB would reduce discomfort, improve maternal satisfaction.

In this system review, as local anesthetic, ropivacaine was used to maintain epidural anesthesia. From the 
A

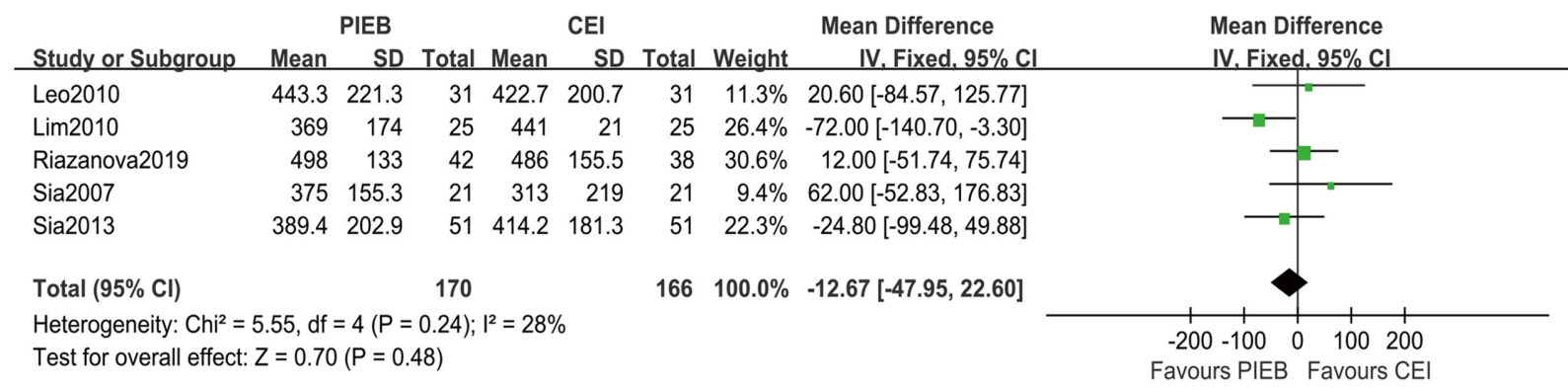

B

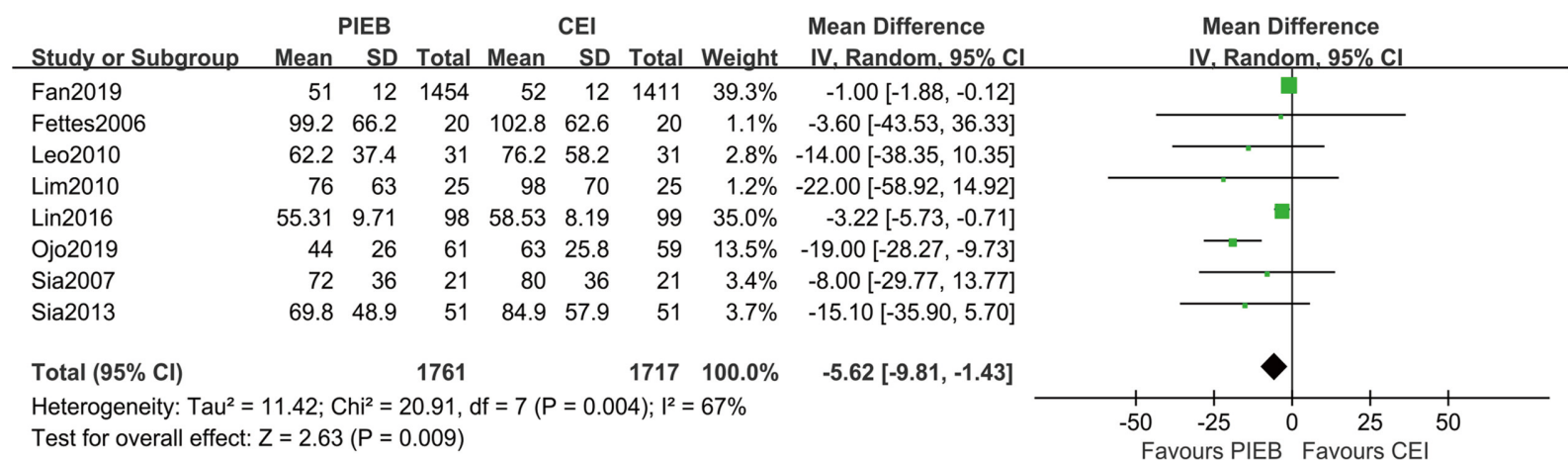

Figure 5 Forest plots of the time of labor. (A) Forest plot of total duration of labor (minute); (B) forest plot of second stage of labor (minute). PIEB, programmed intermittent epidural boluses; CEI, continuous epidural infusion.

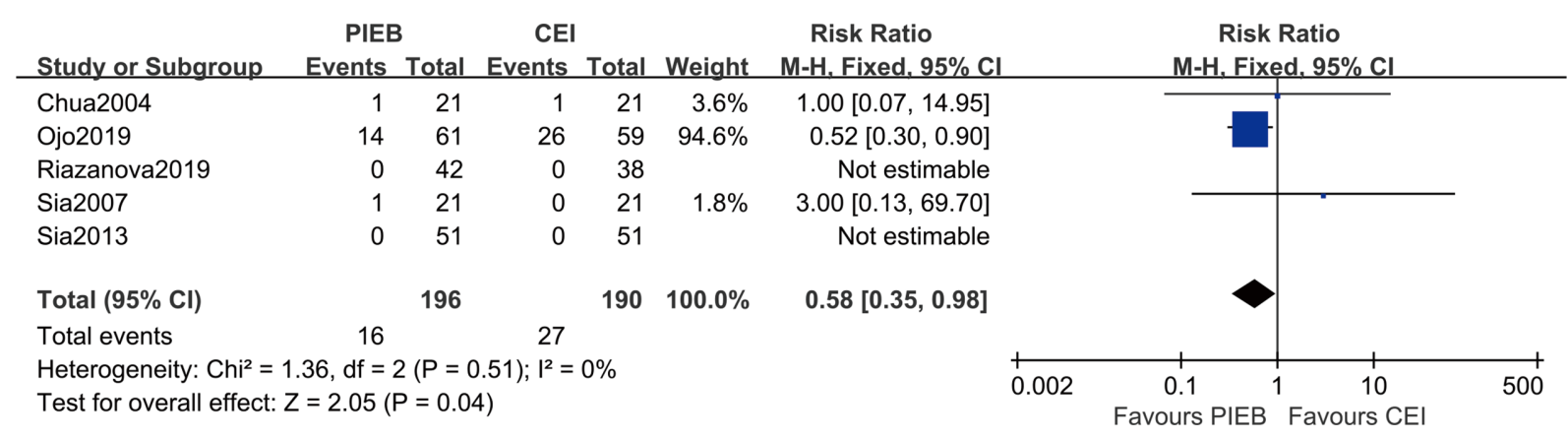

Figure 6 Forest plot of motor block. PIEB, programmed intermittent epidural boluses; CEI, continuous epidural infusion.

result we combined, significant differences were found in consumption of ropivacaine per hour and total. PIEB can achieve satisfied anesthetic effect by less dose of ropivacaine. CEI regime may exceed the most appropriate dose. Extra anesthetic is a burden for maternity and may result in more adverse reaction. Therefore, the lower consumption of ropivacaine is benefit for parturient. But apparent heterogeneity was observed, thus a subgroup analysis was conducted. The subgroup of CSE showed an apparently lower heterogeneity among those trials while a higher heterogeneity was still found in the subgroup with EA. Different ways to maintain labor analgesia might have influenced the consumption. Subgroup analysis was performed and suggested that heterogeneity was especially obvious when using epidural anesthesia in labor analgesia, likely due to the different concentration, volume of ropivacaine. Pregnant women with different weights and ages even could have impact on the spread of ropivacaine. 
A

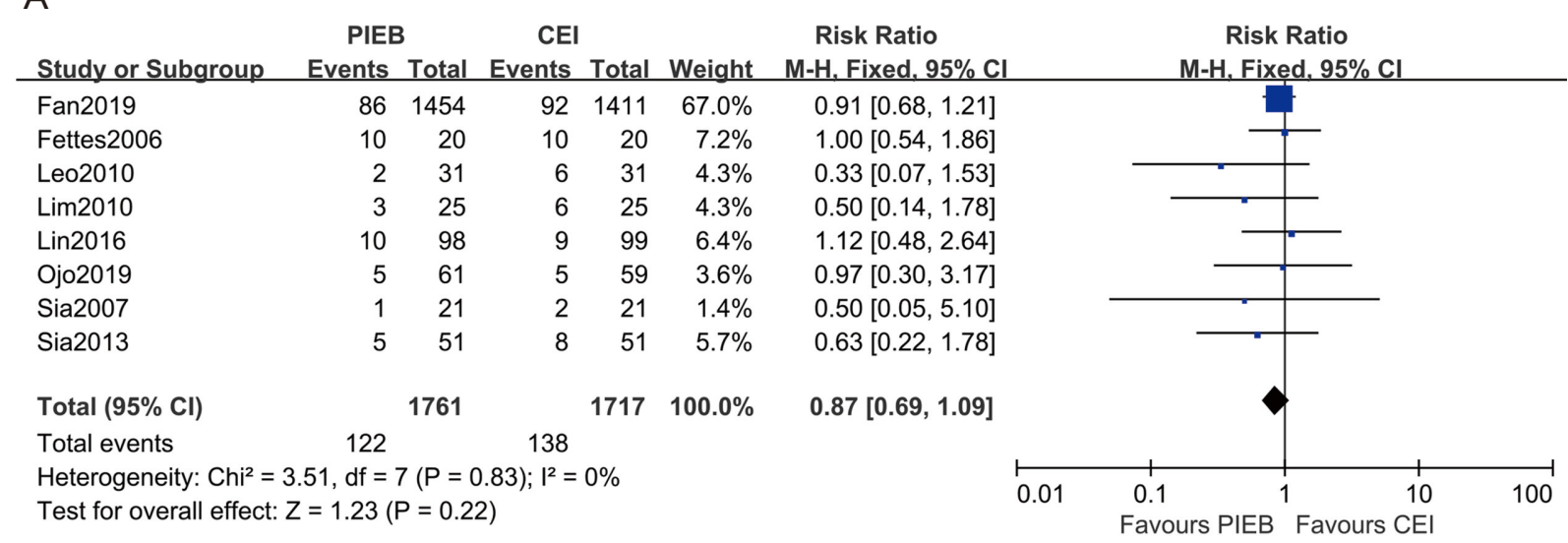

B

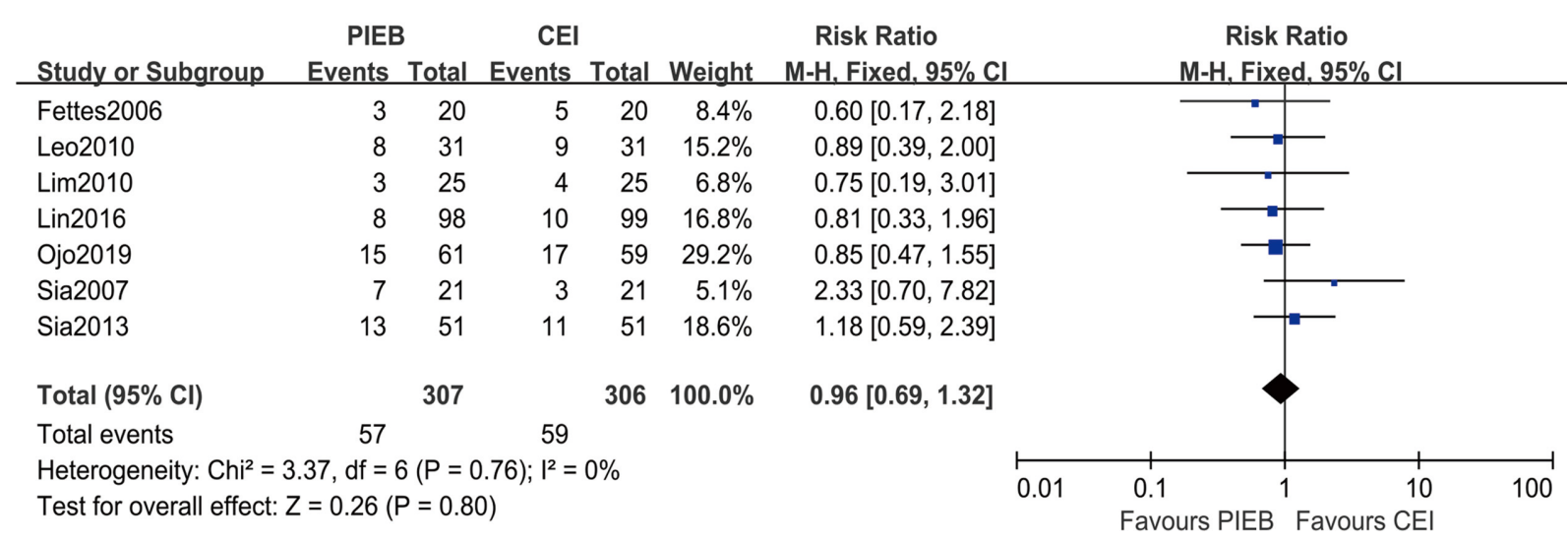

Figure 7 Forest plots of the mode delivery. (A) Forest plot of the mode delivery (instrumental); (B) forest plot of the mode delivery (cesarean). PIEB, programmed intermittent epidural boluses; CEI, continuous epidural infusion.

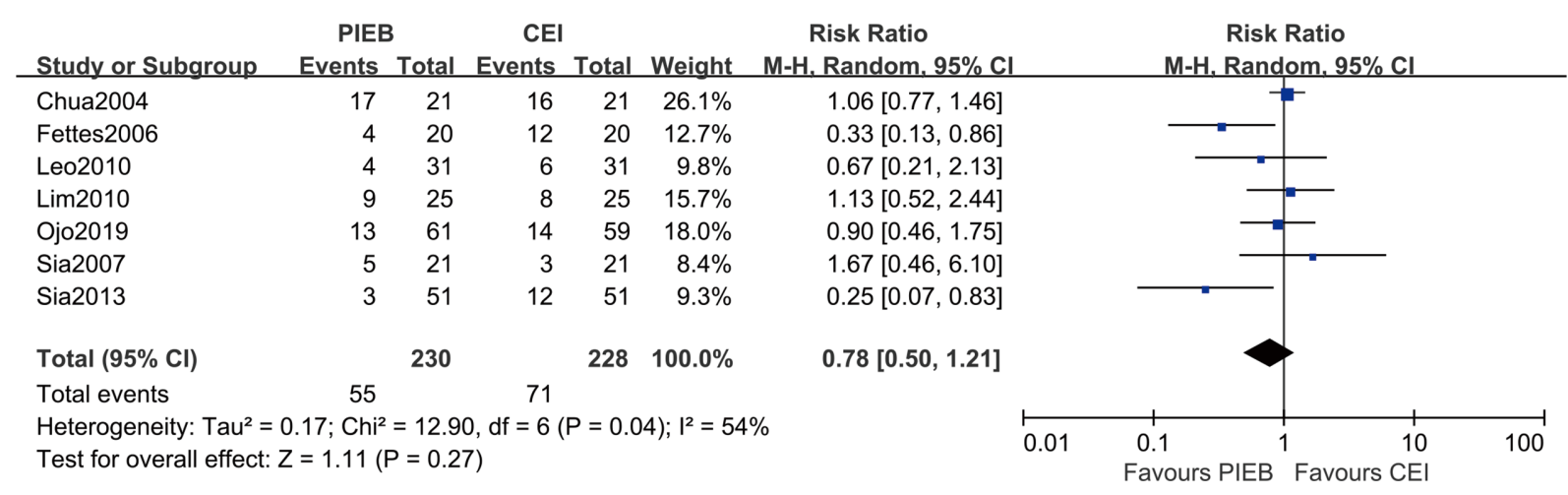

Figure 8 Forest plot of rescue anesthesia. PIEB, programmed intermittent epidural boluses; CEI, continuous epidural infusion.

Thus, all of which may cause heterogeneity. Therefore, the pooled results have to be interpreted with our cautions.

As a matter of fact, Besides the degree of pain, duration of labor and outcomes of fetus are always the most important things that puerpera focus about. Length of labor duration is one of the most intuitive feelings to women in the process 
Table 2 Additional outcomes

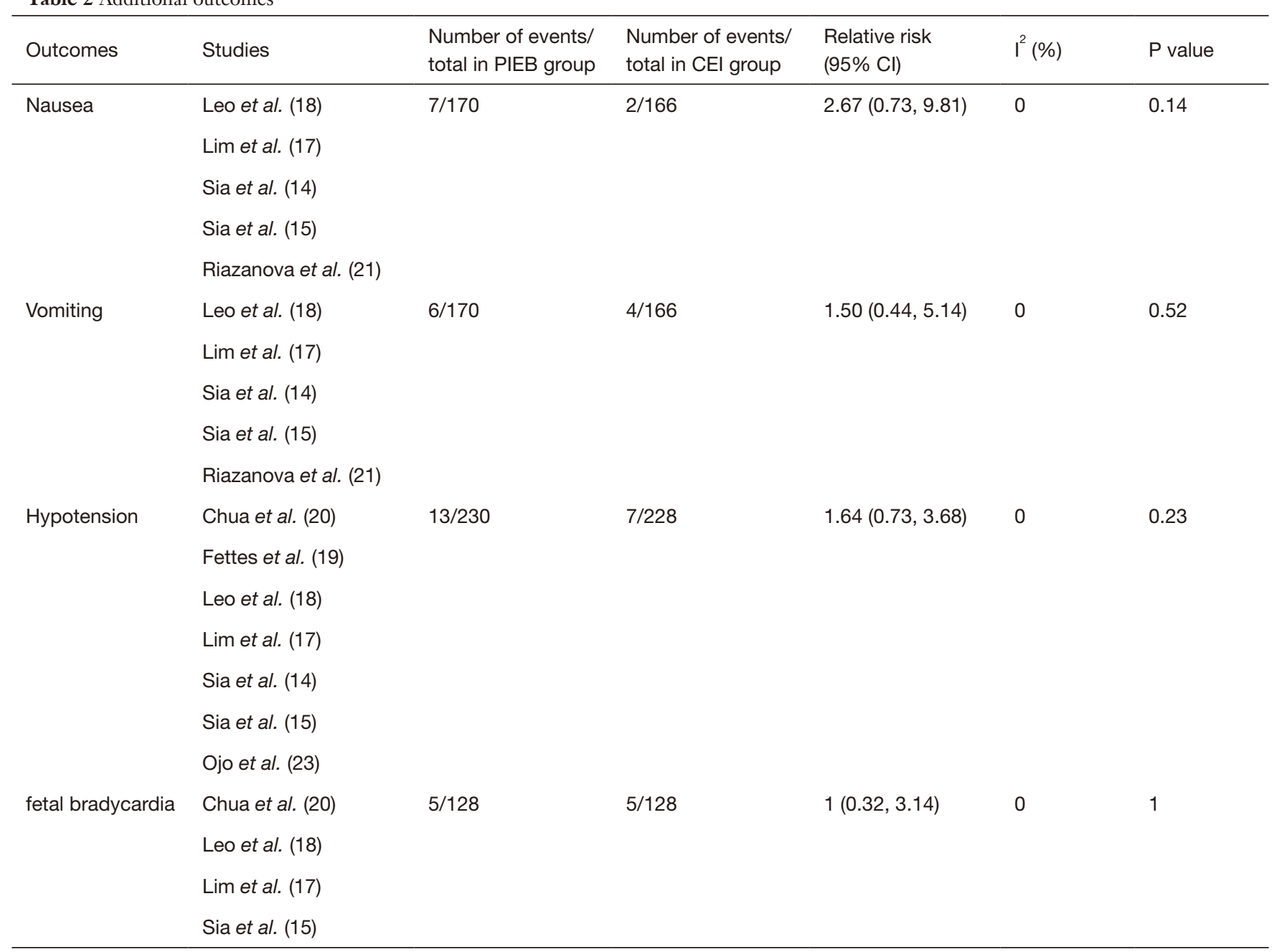

PIEB, programmed intermittent epidural bolus; CEI, continuous epidural infusion.

of childbirth (28). And the duration of second stage of labor is associated with the safety of fetus. According to the data we analyzed, the second stage of labor of PIEB group was shorter than CEI group. Neuraxial analgesia reduces the mobility of patients and pelvic muscle tone, which may impair the ability of "bear down", potentially prolong the duration of second stage of labor (29). More ropivacaine in CEI may result in lower pelvic muscle tone and aggravate block of "bear down" compared with PIEB, and affect the duration of second stage of labor. Thus, PIEB reduces the second stage of labor without increasing the total duration of labor. Also, the reduction of it in PIEB group may improve the outcomes of fetus. Additionally, we couldn't find difference in the rate of fetal bradycardia and Apgar scores in our investigation. The evidence suggests that the safety of fetus in PIEB program is similar to CEI, and PIEB will not increase adverse effect on fetus. Extra indicators of safety on fetus are expected to present in future study to support the finding.

In a published research of Capogna et al. (30), they suggested that lower incidence of motor blockade was associated with a lower occurrence of operative vaginal in PIEB group. Another study (31) also indicated that PIEB reduced the incidence of instrumental delivery compared to CEI regime. But in this study as well as the 2013 systematic review by George et al. (6), we all did not find a significant difference in combined result. In our study, we found that PIEB significantly reduced the probability of motor block. Interestingly, the mode of delivery was similar between two type of techniques according to the pooled 
result. It is reported that ropivacaine has the advantage of the separation of the motor block and sensory block, and may have a short time to recovery of motor function (32). Though, more consumption of ropivacaine resulted in more motor block, the dosage of it is small and the time to recovery of motor function is short, which may not affect the mode of delivery. Therefore, PIEB reduced the incidence of motor blockade but the rate of instrumental vagina delivery has no significant difference but all in low probability. We can conclude that PIEB is as safe as CEI and wouldn't increase the incidence of instrumental and cesarean delivery. As for rescue bolus and other side effect, they were similar in two groups too, which showed that PIEB is the same as CEI in safety.

Limitation of this study should be presented as following. Firstly, sample size of all including studies were small which may not lead to a statistically significant potential valuable clinical outcome. Secondly, different criteria and scales for the judgment were considered as the main source of heterogeneity in the pooling results. And different ways of the evaluation might also cause the heterogeneity. Thirdly, concentration and usage of drugs were not consistent in all trials, and the similar but not identical program of the interventions also affected the final result. Finally, there was no trial reported all outcomes.

\section{Conclusions}

In summary, PIEB show great association with higher maternal satisfaction, reduction of usage of ropivacaine hourly and total as well as shorter duration of second stage of labor without increasing total duration of labor compared to CEI by using ropivacaine to maintain epidural anesthesia. PIEB also decreased the incidence of motor block without increasing other side effects compared with CEI. The results indicated that PIEB is as safe as CEI for maternity and newborns. The number of relative articles is small. Large, high quality, randomized controlled trials are needed for further investigation. Further researches should be aimed to explore the best concentration of ropivacaine and the best interval, volume of bolus to achieve the optimal analgesia.

\section{Acknowledgments}

Funding: This work was supported by awards from National Clinical Key Specialty Construction Project of Ministry of Health [Ministry of Finance (2011) No. 170]; Medical Key
Discipline Construction Project of Chongqing Province [Chongqing Health Science and Education (2007) No. 2].

\section{Footnote}

Reporting Checklist: The authors have completed the PRISMA reporting checklist. Available at http://dx.doi. org/10.21037/apm-20-1541

Conflicts of Interest: All authors have completed the ICMJE uniform disclosure form (available at http://dx.doi. org/10.21037/apm-20-1541). The authors have no conflicts of interest to declare.

Ethical Statement: The authors are accountable for all aspects of the work in ensuring that questions related to the accuracy or integrity of any part of the work are appropriately investigated and resolved. The data in this paper is from the database, so ethical approval and informed consent are not required.

Open Access Statement: This is an Open Access article distributed in accordance with the Creative Commons Attribution-NonCommercial-NoDerivs 4.0 International License (CC BY-NC-ND 4.0), which permits the noncommercial replication and distribution of the article with the strict proviso that no changes or edits are made and the original work is properly cited (including links to both the formal publication through the relevant DOI and the license). See: https://creativecommons.org/licenses/by-nc-nd/4.0/.

\section{References}

1. Melzack R. The myth of painless childbirth (the John J. Bonica lecture). Pain 1984;19:321-37.

2. Lavand'homme P. Chronic pain after vaginal and cesarean delivery: a reality questioning our daily practice of obstetric anesthesia. Int J Obstet Anesth 2010;19:1-2.

3. Tan HS, Sng BL, Sia ATH. Reducing breakthrough pain during labour epidural analgesia: an update. Curr Opin Anaesthesiol 2019;32:307-14.

4. Sng BL, Sia ATH. Maintenance of epidural labour analgesia: The old, the new and the future. Best Pract Res Clin Anaesthesiol 2017;31:15-22.

5. Wang Q, Zheng SX, Ni YF, et al. The effect of labor epidural analgesia on maternal-fetal outcomes: a retrospective cohort study. Arch Gynecol Obstet 2018;298:89-96. 
6. George RB, Allen TK, Habib AS. Intermittent epidural bolus compared with continuous epidural infusions for labor analgesia: a systematic review and meta-analysis. Anesth Analg 2013;116:133-44.

7. Wong CA, Scavone BM, Peaceman AM, et al. The risk of cesarean delivery with neuraxial analgesia given early versus late in labor. N Engl J Med 2005;352:655-65.

8. McKenzie CP, Cobb B, Riley ET, et al. Programmed intermittent epidural boluses for maintenance of labor analgesia: an impact study. Int J Obstet Anesth 2016;26:32-8.

9. Carvalho B, George RB, Cobb B, et al. Implementation of Programmed Intermittent Epidural Bolus for the Maintenance of Labor Analgesia. Anesth Analg 2016;123:965-71.

10. Ateser RY, Kayacan N. Intrathecal ropivacaine in cesarean delivery. Niger J Clin Pract 2017;20:1322-7.

11. Polley LS, Columb MO, Naughton NN, et al. Relative analgesic potencies of levobupivacaine and ropivacaine for epidural analgesia in labor. Anesthesiology 2003;99:1354-8.

12. Savović J, Weeks L, Sterne JA, et al. Evaluation of the Cochrane Collaboration's tool for assessing the risk of bias in randomized trials: focus groups, online survey, proposed recommendations and their implementation. Syst Rev 2014;3:37.

13. Hozo SP, Djulbegovic B, Hozo I. Estimating the mean and variance from the median, range, and the size of a sample. BMC Med Res Methodol 2005;5:13.

14. Sia AT, Lim Y, Ocampo C. A comparison of a basal infusion with automated mandatory boluses in parturientcontrolled epidural analgesia during labor. Anesth Analg 2007;104:673-8.

15. Sia AT, Leo S, Ocampo CE. A randomised comparison of variable-frequency automated mandatory boluses with a basal infusion for patient-controlled epidural analgesia during labour and delivery. Anaesthesia 2013;68:267-75.

16. Lin Y, Li Q, Liu J, et al. Comparison of continuous epidural infusion and programmed intermittent epidural bolus in labor analgesia. Ther Clin Risk Manag 2016;12:1107-12.

17. Lim Y, Chakravarty S, Ocampo CE, et al. Comparison of automated intermittent low volume bolus with continuous infusion for labour epidural analgesia. Anaesth Intensive Care 2010;38:894-9.

18. Leo S, Ocampo CE, Lim Y, et al. A randomized comparison of automated intermittent mandatory boluses with a basal infusion in combination with patient- controlled epidural analgesia for labor and delivery. Int J Obstet Anesth 2010;19:357-64.

19. Fettes PD, Moore CS, Whiteside JB, et al. Intermittent vs continuous administration of epidural ropivacaine with fentanyl for analgesia during labour. Br J Anaesth 2006;97:359-64.

20. Chua SM, Sia AT. Automated intermittent epidural boluses improve analgesia induced by intrathecal fentanyl during labour. Can J Anaesth 2004;51:581-5.

21. Riazanova OV, Alexandrovich YS, Guseva YV, et al. A randomized comparison of low dose ropivacaine programmed intermittent epidural bolus with continuous epidural infusion for labour analgesia. Rom J Anaesth Intensive Care 2019;26:25-30.

22. Fan Y, Hou W, Feng S, et al. Programmed intermittent epidural bolus decreases the incidence of intra-partum fever for labor analgesia in primiparous women: a randomized controlled study. Arch Gynecol Obstet 2019;300:1551-7.

23. Ojo OA, Mehdiratta JE, Gamez BH, et al. Comparison of Programmed Intermittent Epidural Boluses With Continuous Epidural Infusion for the Maintenance of Labor Analgesia: A Randomized, Controlled, DoubleBlind Study. Anesth Analg 2020;130:426-35.

24. Craig D, Carli F. Bromage motor blockade score - a score that has lasted more than a lifetime. Can J Anaesth 2018;65:837-8.

25. Angle P, Landy CK, Charles C, et al. Phase 1 development of an index to measure the quality of neuraxial labour analgesia: exploring the perspectives of childbearing women. Can J Anaesth 2010;57:468-78.

26. Sng BL, Zeng Y, de Souza NNA, et al. Automated mandatory bolus versus basal infusion for maintenance of epidural analgesia in labour. Cochrane Database Syst Rev 2018;5:CD011344.

27. Akca A, Corbacioglu Esmer A, Ozyurek ES, et al. The influence of the systematic birth preparation program on childbirth satisfaction. Arch Gynecol Obstet 2017;295:1127-33.

28. Petersen A, Penz SM, Gross MM. Women's perception of the onset of labour and epidural analgesia: a prospective study. Midwifery 2013;29:284-93.

29. Thornton JG, Capogna G. Reducing likelihood of instrumental delivery with epidural anaesthesia. Lancet 2001;358:2.

30. Capogna G, Camorcia M, Stirparo S, et al. Programmed intermittent epidural bolus versus continuous epidural infusion for labor analgesia: the effects on maternal motor 
function and labor outcome. A randomized double-blind study in nulliparous women. Anesth Analg 2011;113:82631.

31. Xu J, Zhou J, Xiao H, et al. A Systematic Review and Meta-Analysis Comparing Programmed Intermittent Bolus and Continuous Infusion as the Background

Cite this article as: Huang $\mathrm{R}, \mathrm{Zhu} \mathrm{J}$, Zhao Z, Wang B. The effect of programmed intermittent epidural bolus compared with continuous epidural infusion in labor analgesia with ropivacaine: a meta-analysis of randomized controlled trials. Ann Palliat Med 2021;10(3):2408-2420. doi: 10.21037/apm-20-1541
Infusion for Parturient-Controlled Epidural Analgesia. Sci Rep 2019;9:2583.

32. Malhotra R, Johnstone C, Halpern S, et al. Duration of motor block with intrathecal ropivacaine versus bupivacaine for caesarean section: a meta-analysis. Int J Obstet Anesth 2016;27:9-16. 
1. pregnancy [Title/Abstract]

2. parturient [Title/Abstract]

3. delivery [Title/Abstract]

4. obstetric [Title/Abstract]

5. labor [Title/Abstract]

6. labour [Title/Abstract]

7. obstetrical [Title/Abstract]

8. delivery, obstetric"[Mesh]

9. "labor, obstetric"[Mesh]

10. "obstetrics"[Mesh]

11. 1 OR 2 OR 3 OR 4 OR 5 OR 6 OR 7 OR 8 OR 9 OR 10

12. automated intermittent epidural bolus [Title/Abstract]

13. automated mandatory boluses [Title/Abstract]

14. automated intermittent mandatory boluses [Title/ Abstract]

15. programmed intermittent bolus [Title/Abstract]

16. intermittent epidural bolus [Title/Abstract]

17. programmed intermittent epidural bolus [Title/
Abstract]

18. PIEB [Title/Abstract]

19. Intermittent [Title/Abstract]

20. epidural [Title/Abstract]

21. 19 AND 20

22. 12 OR 13 OR 14 OR 15 OR 16 OR 17 OR 18 OR 21

23. ropivacaine [Title/Abstract]

24. ropivacaine hydrochloride[Title/Abstract]

25. 23 AND 24

26. randomized controlled trial[Title/Abstract]

27. clinical trials [Title/Abstract]

28. random allocation [Title/Abstract]

29. randomized*[Title/Abstract]

30. "random allocation"[Mesh]

31. "randomized controlled trials as topic"[Mesh]

32. "controlled clinical trial" [Publication Type]

33. "randomized controlled trial" [Publication Type]

34. 26 OR 27 OR 28 OR 29 OR 30 OR 31 OR 31 OR 33

35. 11 AND 22 AND 25 AND 34

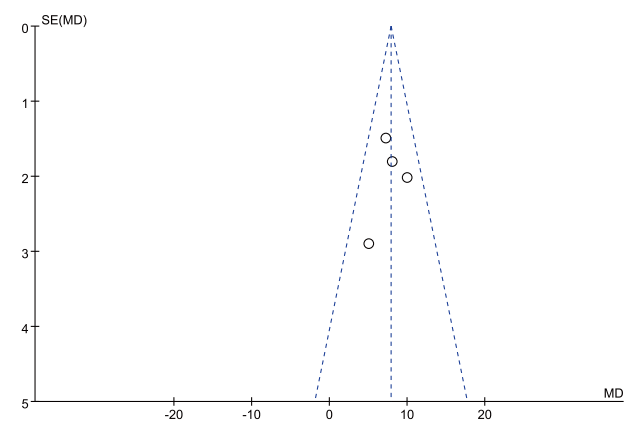

Figure S1 Funnel plot. 